\title{
Using Observation and Refinement to Improve Distributed Systems Test
}

\author{
Johan Moe \\ Ericsson $A B$ \\ Box 1248 \\ SE-581 12 Linköping, Sweden \\ +4613-284894 \\ johan.moe@era.ericsson.se
}

\author{
David A. Carr \\ Institutionen för Systemteknik \\ Luleå Tekniska Universitet \\ SE-971 87 Luleå, Sweden \\ +46920-491965 \\ david@sm.luth.se
}

\author{
Mikael Patel \\ Ericsson $A B$ \\ Box 1248 \\ SE-581 12 Linköping, Sweden \\ +46 13-28 4665 \\ mikael.patel@era.ericsson.se
}

\begin{abstract}
Testing a distributed system is difficult. Good testing depends on both skill and understanding the system under test. We have developed a method to observe the system at the CORBA remote-procedure-call level and then use dynamic-query-based visualization to refine and improve the test cases. The method and accompanying tools have been tested and refined by using them as part of the software support effort for two distributed application, each having about $500 \mathrm{~K}$ lines of code. During this time the tools have been adapted to support testing by adding a scripting mechanism that permits the visualization tool to specify test reports. We also added parameter value observation and reporting. Finally, we added an active probing mechanism to induce faults and delays in order to stress the system under test. Our efforts have led to a substantial improvement in system test quality.
\end{abstract}

\section{Introduction}

Testing a software system is difficult and requires skill [9]. Systems testers must validate all requirements. In order to fully describe the fault model, they need to understand the system to be tested and its runtime environment. Technological and architectural decisions will equire additional testing often based on structural system information. Testers must have a good command of many techniques. They must write effective test cases but still select a meaningful subset of all possible test cases in order reduce test size to something that is tractable.

Distributed systems make the task more difficult. For better performance, parallel execution is introduced and with that the possibility of race conditions. Communication time is not guaranteed, and it is difficult to foresee how performance on a user level is affected by low-level communication. Clearly, testers need training about distributed systems. In addition, testers need new tools and methods in order to bserve and understand distributed system behavior.

We are developing a method designed to improve a tester's understanding of the behavior of distributed sys- tems that they test. In daily work, the method also guides testers as they look for suspected malfunctions or seek to improve their test cases. Finally, the method can be used to evaluate system test quality.

The method (Figure 1) can be seen as a loop over a number of steps:

- Observation,

- Analysis and calculation of statistics,

- Visualization (with drilling down for extra details),

- System or test modification,

- Repeat until satisfied.

The tools supporting the method use CORBA interceptors [21] to intercept remote procedure calls between clients and servers. The intercepted data is then processed using a custom program to create logical events (such as matching calls and returns) and compute summa ry statistics (behavioral information such as call frequencies, exceptions, and load). The summary statistics are then vis ualized using an extended version of Stardom [4], a vis ualization tool based on dynamic queries [1]. The information from the statistics and visualization are used to discover system errors and refine test cases. Finally, the procedure is repeated as necessary.

Our goal is to build methods that will be used in commercial software testing. Therefore, we have been refining our method by using it in the testing efforts for two products with about $500 \mathrm{~K}$ lines of code in each. We be-

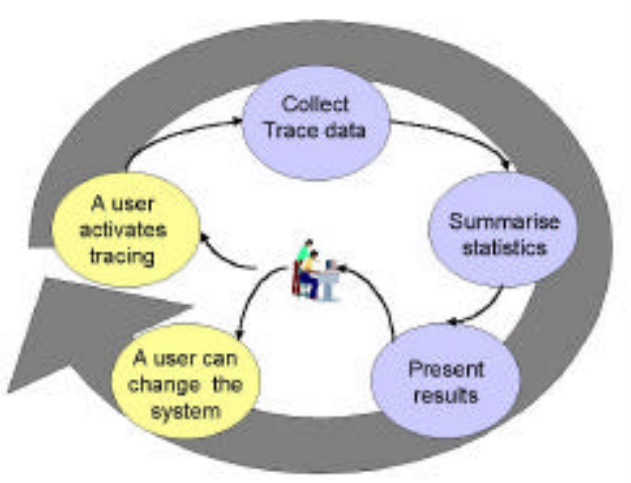

Figure 1 - a schematic view of the method. 
gan by using the method to support the Radio Network Optimization (RNO) platform and subsequently began to support the Common Integration Framework (CIF) system. Our current method is based on both features that worked well in RNO directly and fe atures that are refined or developed with CIF. While refining our method, we have interviewed testers, passively observed their workgroups, and at times become active in their daily work.

Our method has been motivated by a need within our organization for improved system testing. First contacts with managers often elicited a comment like "...we have so many tools, but we don't know what we've tested...". Although the organization kept track of requirements and their test cases, more experienced people felt that the system was not adequately tested. We thought that a logical first step would be to measure remote procedure call coverage. With this we can get a picture of how well test cases execute the system regardless of the requirements or other specifications.

Results using the method have been promising. Testers have built new test cases and improved old ones. They and their managers now have quantitative data for evaluating test quality.

The next section of this paper gives a quick overview of the related work. This will be followed by a brief overview of our method with examples of its use during test execution. Next, we will discuss the results and implications of the method's use within our organization. Finally, we will summarize and discuss future work.

\section{Related Work}

Our research draws on work in three related areas: software observation, testing, and software visualization.

\subsection{Software observation}

Our approach is similar to the approach used by some commercial observation tools, e.g. Application Response Measurement (ARM) from the Computer Measurement Group [6]. ARM is an API that makes it possible for applications to log information for analysis by commercial ARM compatible tools. However, ARM requires source code modification or using a remote terminal emulator that limits monitoring to user initiated transactions. For exis ting systems such as the RNO and CIF, modifying the source is too costly, and using a remote terminal emulator will not provide sufficiently detailed information.

Observation approaches can be divided into macroscopic and microscopic [7]. Macroscopic approaches collect cumulative information while microscopic approaches, often called tracing, concentrate on sequences of messages. We often find macroscopic tools regularly running in an operational environment. With these tools, unwanted behavior can be detected. When a fault is spot- ted or suspected, more detail is usually desired, and a microscopic tool is employed.

Our method should be seen as macroscopic when it comes to visualization. It is similar to the object-oriented macroscopic visualization of R. J. Walker, et al. [[24] and W. De Pauw, et al. [7]. However, we are not focused on object-oriented systems but on large distributed systems. We have found that as observation sessions grow longer, one can perceive the stream of observation data as infinite. Therefore, we present information summary statistics by time period (per hour, etc.). This is in order to build detailed operational profiles [20] that can be used to evaluate test case "realism" and to guide their refinement.

One uses microscopic approaches to learn how something is accomplished or to get more detail. De Pauw's [7] work focuses on displaying execution traces in an objectoriented system. They use Jacobson's interaction diagram [11] as a basis for visualizing execution sequences. They introduce a modific ation to the diagram to make recursion explicit and introduce several methods to compress the diagram. By contrast, our work concentrates on summary statistics of observation data over (relatively) long periods of time and uses scatter plot presentations of these statistics.

Artificially inserting, deleting, or slowing messages is called active probing. It has been used to study the behavior of TCP [5]. We have adapted this idea to our observation environment by adding the ability to delay server response and throw exceptions. In this way, we can "stress test" software without requiring large additional resources. Similar ideas for network performance discovery are presented in [23].

\subsection{Testing (and detection)}

Ghosh and Mathur [10] proposed a number of quality measures for distributed, component -based, system's testing, with special focus on CORBA. They discuss the importance of having coverage measures on method, exception, and parameter levels in order to obtain high quality testing. Coverage measurement also allows measuring reliability or predicting the number of remaining defects. $\mathrm{Li}$, et. al. [14] demo nstrate that the number of faults found taken together with the degree of coverage can predict the remaining faults. They claim that broader coverage, such as branch coverage, is better than narrower coverage, such as statement coverage. In this sense, method, exception, and parameter coverage for CORBA servers are too narrow. However, it is often the best that can be achieved, particularly for off-the-shelf components.

There are alternatives to coverage for test quality evaluation. "Jester" for Java [19] changes the source code by injecting faults and only accepts the test suite as good if these faults are discovered. However, source code is not always available, which renders this approach impossible. 
Automatic fault detection has been tried by a number of researchers. For microscopic approaches, automatic loop detection has been tried in De Pauw, et al. [7] and J. R. Larus [13]. A totally different approach to fault detection is the use of query languages such as PARFORMAN [2]. With this language the authors demonstrate approaches to race detection, deadlock, and other concurrent programming problems. We also allow the tester to build queries. However, our queries are built graphically by visualizing in Stardom and then writing the parameter settings as a script that is applied against future observations.

\subsection{Visualization}

In contrast to our approach of visualizing dynamic events, software visualization has traditionally concentrated on the source code as a basis for the visualization. Various metaphors have been used in order to increase understanding. SeeSoft [8] used a source-file-based metaphor with files represented by boxes and with source code lines represented by colored lines within the boxes. SeeSoft provided visualization of test coverage although it emphasized dis playing source-code-modification history.

The call graph and class diagram have also been used as a basis for software visualization. Recent examples include C++ Lens [3], Software World [12], and Schauer and Keller's pattern-based tool [22]. The C++ Lens displays inheritance hierarchies as trees and uses size encoding to emphasize relationships such as the number of re ferences. Software World uses a cities-neighborhoodsbuildings metaphor to represent software inheritance and containment relationships. Schauer and Keller's tool uses a network to display interclass relationships with design patterns as an organizing theme.
Source-code-based visualizations primarily emphasize static aspects of software. Walker, et al. [24] report on a trace visualization system that concentrates on dynamic aspects of object-oriented systems. Their system uses animation to convey dynamic relationships, and boxes and arrows to convey static relationships.

None of the above systems provide real-time, dynamic filtering as is available with dynamic queries [1]. Some, such as SeeSoft, allow users to customize their displays.

\section{The Method B asics}

Most of the method's details have been described in [16, 17, 18]; however, a brief description of the observation and vis ualization follows.

\subsection{Observation and Calculating Statistics}

Observed execution data is the basic input used in the method. Using observation in a commercial setting equires low impact on system performance and minimal extra work for developers. Our implementation supports Java and C++ under two different CORBA implementations, Borland's Visibroker and Iona's Orbix. Calls to a server are intercepted, and the complete message buffer (object reference, method and actual parameters) is captured and sent to a log server together with performance measures. Most statistics are calculated during postprocessing in order to minimize run-time impact.

Parameter handling has recently been added, and in addition to coverage, it is possible to calculate statistics on user-defined equivalence classes.

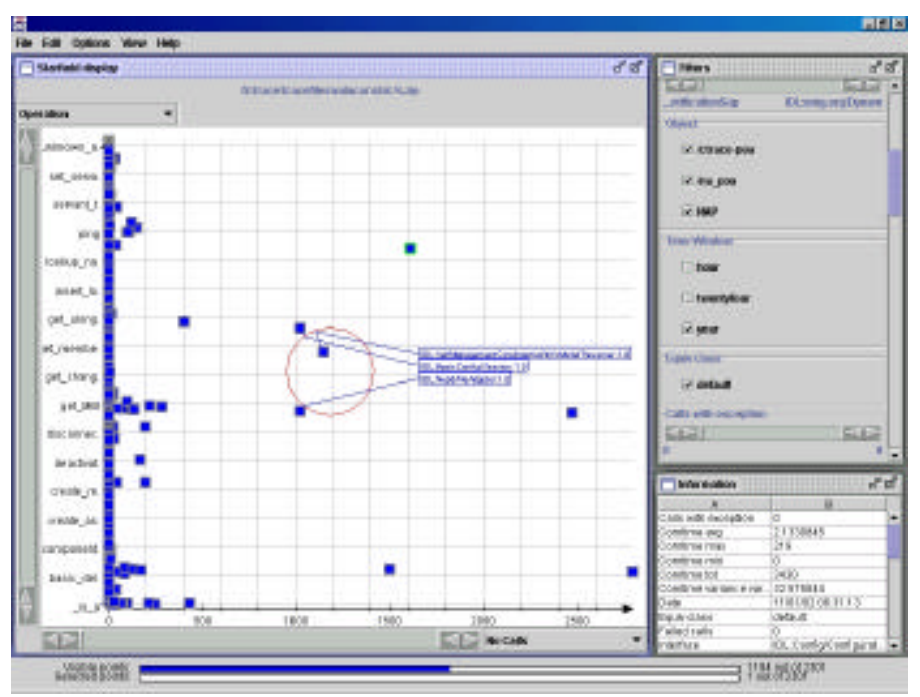

Figure 2 -The default view when Stardom is started. 


\subsection{Visualizing Testing Results}

We are currently using a customized version of the Stardom [4] visualization tool to analyze the summary statistics of the observation. Stardom supports rapidly constructing a scatter plot of data sets. It also allows specifying and changing the graphical encoding of parameters.

For example, a test analyst may be interested in how many times each method has been called. This is displayed by choosing number-of-calls and method as the $\mathrm{x}$ and $y$-axes. Other parameters can be configured for presentation on the graph as mark shape, color, size, etc. Stardom also supports real-time filtering using widgets for specifying the ranges and values. This technique is called "dynamic queries" [1].

Stardom and its data display do not contain complex visual elements, so learning time is minimal. Its power is in filtering and the real-time response to filter changes. This permits simply and rapidly setting up a custom view to study an aspect of system behavior. For example, a custom view to investigate coverage can be constructed as follows:

- Figure 2 shows the default view in Stardom when the observation data is imported - operations, given by the IDL interfaces, versus number-of-calls for each.

- In order to display all interfaces and their uncalled operations, the analyst selects the drop-down menu for the parameter to be displayed on the $\mathrm{x}$-axis and selects "interfaces". Next, the analyst sets " 0 " for the numberof-calls using the filter pane (on the right side). The display now shows a square for all uncalled methods by interface. If desired, analysts can reduce display clutter by selecting only those interfaces or methods that they deem interesting. Figure 3 comes from actual system testing and shows many uncalled methods.

In order to further automate the analysis, you can cre- ate a "report" from current filter settings. The analyst could save this vie $w$ as a report and then access it with a single click for any future observation. The analyst may also elect to generate a script that runs directly on a su mmarized observation file. This can be used to present the same plot as text rows in a terminal window. When run as post processing after a test, the script can be use to list all uncalled methods.

After including all the uncalled methods in test cases, the analyst may want to focus on parameter coverage [10]. While more involved than method coverage, the following example illustrates the investigation of parameter coverage.

- From the set up of the previous example, analysts change the number-of-calls filter to be greater than zero. As the dis play is already limited to interfaces of interest, analyst see squares for all called, interesting methods.

- Analysts must now examine each method individually. They do this by "drilling-down" for details. After deciding to investigate a specific method, say "create_session", an analyst would select the square associated with that method and choose parameters from the pop-up menu. Information about the parameter values would then appear in a text -editor window.

- As test analysts are likely to be uncertain about parameter syntax and semantics, they can also drilldown to the IDL-specification, which appears in another editor window (Figure 4). They could then use this information to make decisions about parameter coverage. For example, Figure 4 shows that parameter values for timeouts are set to $-1,60,000$, and 300,000 . From the IDL file, one can see that the units are milliseconds and -1 means an infinite timeout. An analyst could decide that this was inadequate and test 0 and 1 seconds as well.

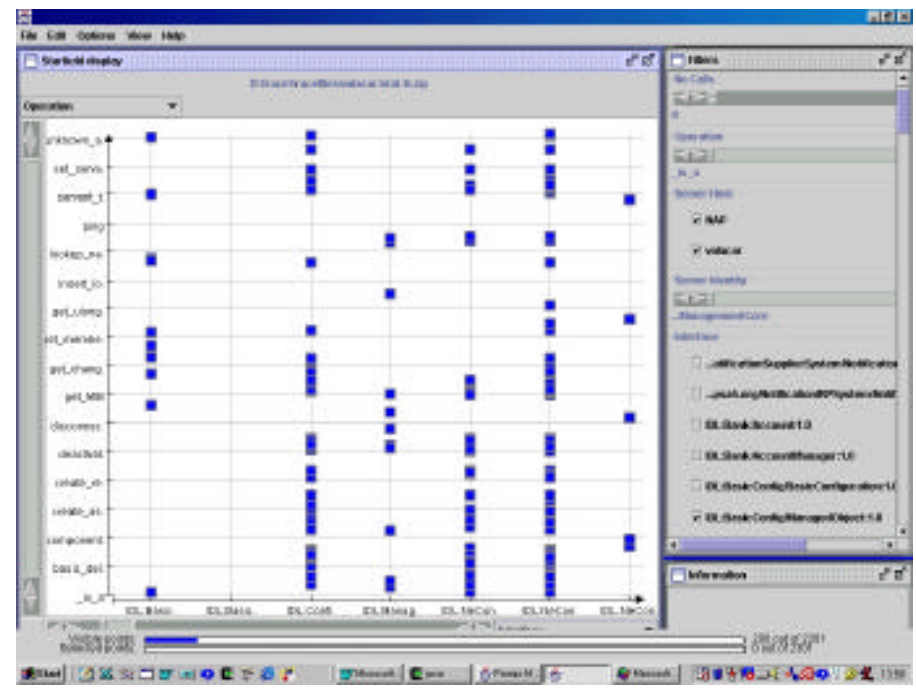

Figure 3-A view of uncalled methods for the IDL:BasicConfig/ManagedObject:1.0 interface 


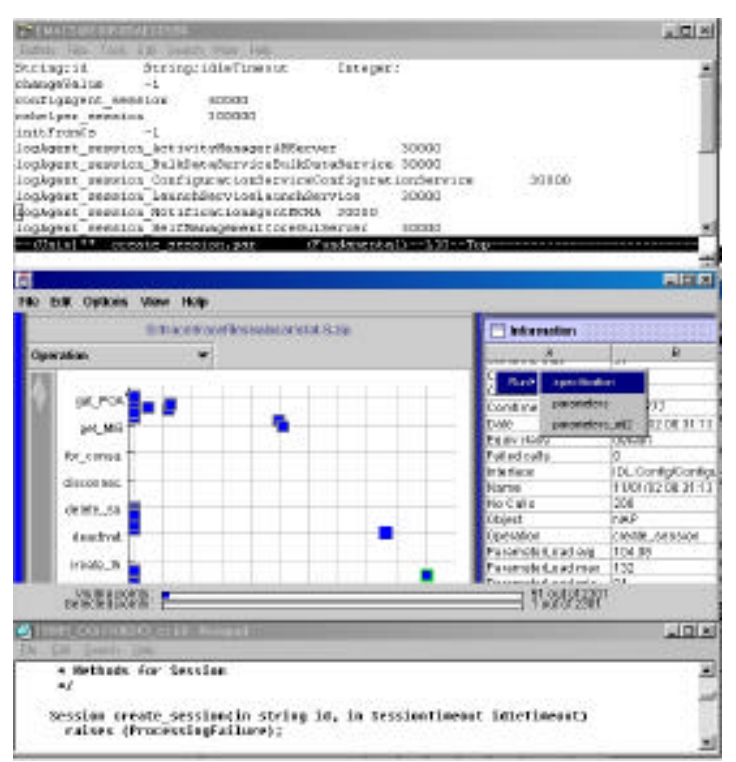

Figure 4-Parameter values (top), visualization (middle), and IDL (bottom).

As one can see from the above description, the immediate visual feedback encourages analysts to explore the data. The dynamic queries method in effect allows analysts to perform dozens of queries per minute by changing filters and by changing the parameters that are displayed on each axis. In addition, a standard visualization tool with simple visual displays is in keeping with our philosophy of simplicity so that developers don't spend much time learning to use the sy stem.

\section{Discussion}

We have been using and refining our method for about two years within software development organizations at Ericsson. We began with RNO in the software maintenance group. Our experience within that group led us to extend its use to RNO testers. As the method stabilized and its value became apparent, we wanted to broaden the user base and make the method more general. Thus, we expanded to include CIF about a year ago.

Along the way, we learned much about supporting system test. First, testers require special automation support from their tools. Second, test improvement requires much more than improving test coverage. For customers, system performance is important, and testing should provide a simulation of their operations. Error conditions and slower hardware are also difficult to test. For this reason, we added active probing to our observation tool. Finally, the human factor must be considered.

\subsection{Adapting to the Test Environment}

One major shift from the maintenance environment to testing was the need to support "batch mode". Testers want to test automatically and only receive notifications of problems. In order to accommodate this need, we added the scripting facility to Stardom. This enabled test engineers to use the visualization as a kind of query builder or report generator. Testers used scripts for benchmarking, for trend reports, and as simple triggers indicating performance problems.

\subsection{Coverage}

Using coverage visualization from test execution observation, testers found that many interfaces were not adequately tested. Surprisingly, many interfaces were not called at all. Armed with this knowledge, testers were able to provide additional test cases for method coverage.

Once test engineers were able to inspect the IDL definitions and parameter values, they began to construct better parameter coverage. However, deciding what was adequate proved problematic. Therefore, we adopted a simple approach of exhaustively testing enumerated data types as they often direct execution. Similarly, we decided that sequences of length zero, one, and many would be useful test cases. Test cases for integers and strings were not extensively expanded as semantic information is often missing from the IDL definitions. With these guidelines, testers were able to produce better parameter value domains and achieve higher coverage on interfaces, methods, and parameters.

We also added the ability to compute statistics on equivalence classes (value ranges) for individual parameters. However, this feature was not used. After investigation, we found that semantic information was not available in either the IDL or the requirements specification. In order for equivalence class reports to be useful, this information must appear in the system documentation.

On a system design level, inspection of the coverage information led to the discovery that some child interfaces were derived from their parents because they needed a subset of the parent's methods. This can indicate that the system designers did not adequately factor the system in their original design.

\subsection{Coverage and Operational Profiles}

Even the above improvements, do not guarantee realistic testing. Because our observation collection requires few resources, we were able to observe RNO at customer sites during daily operation. It was then possible to make co mparisons between test cases and actual operations. This comparison showed that test case execution statistics differed greatly from customer operations. 


\begin{abstract}
Although the primary objective of RNO and CIF testing was verification, management did want to avoid the embarrassment of delivering a verified system where performance for some function was noticeably poorer than a previous version. By adding tests that more closely match customer usage, testers hope to minimize this risk.
\end{abstract}

\subsection{Active Probing}

Matching customer loads or trying to induce load-related faults can take an enormous amount of resources. In order to add stress testing to the system, we implemented active probing in our observation mechanism. Because CORBA interceptors sit between the server communications software and the server software itself, it is possible to delay or discard remote procedure calls. It becomes possible to induce faults, timeouts, racing conditions (hazards), execution of retry logic or fault tolerance mechanisms, etc.

By delaying all calls, it is possible to observe how delays in one area of the system cascade and affect others. Using this technique, it is possible to test the effect of slower machines or communication lines and evaluate potential customer cost saving measures.

The possibility to raise different exceptions at the server means that one can simulate problems in communication or other situation. Such problems can be difficult to create during system testing.

Using this feature, testers discovered instances where the developers had misunderstood the CORBA execution model. In one case, they found that clients repeatedly called a server when the exception indicated that the server would never be available. In another situation, an induced exception blocked an entire application while a client waited "forever" for an inoperative server to espond. The only "work around" discovered was to restart the client - an unsatisfactory solution for customers.

\subsection{The Human Factor}

Using and refining the tool within the test organizations has been a learning experience for both the testers and the researchers.

For testers, using the tool has been an eye-opening experience. They have improved their understanding of what is actually happening inside of the system. The tool also provided a way to evaluate their own tests, often finding incorrect tests. For example, a test program was supposed to run 10,000 transactions. But, our tool showed the tester that out of the 10,000 transactions, 6,000 were rolled back.

The method also encouraged testers to break from their reactive mode of working. Typically, they run a test suite and look for discrepancies between the specification (or expected results) and the outcome. Any deeper analysis was seen as more of a concern for the developers. Now, test engineers are encouraged to investigate the IDL (previously the developers' domain) and understand the highlevel system architecture.

We also discovered things. First, it became necessary to give a short course in CORBA and client-server systems. Without it, testers could see no need for the tool. We did not at first fully appreciate the differences between testers and developers testing software. When we more fully understood the tester's job, we implemented support for automating inspection of observations. By adding report and query generation to the presentation tool, it became possible to create specific reports. These gave the equivalent of "a green or red light," which indicated to testers that something was okay or wrong.

\section{Conclusion and Future Work}

We have presented a method to improve test quality by analyzing intercepted information at the remoteprocedure-call level. This method involves:

- Observation via CORBA interceptors

- Post processing to compute summary statistics

- Visualization of the statistics using a customized version of the Stardom visualization tool.

The method supports exploration of test results including display of both method and parameter coverage. By supporting a "save view" feature, our version of Stardom allows the test analyst to reuse displays that are found to be useful when exploring the data. Furthermore, the saved view can be exported into a script that can be used to automatically process observed data. By using these two features, testers can create custom reports for inclusion in their test analysis.

We have used and refined this method within the support organizations for two large distributed applications. The method has resulted in improved testing and gives good payback for the effort involve. With the method, testers can evaluate test quality. The method improves testers' comprehension of system execution enabling them to better verify correct execution on system level.

Clearly, the method and exposure to the researchers seems to have had an educational effect on the testers. Before, testers talked about the system and user interfaces. Now, they also talk about the internals (clients and servers) and their communication.

However, there is still much room for improvement. It is clear that the tool does not present parameters and their coverage in a useful way. Analyzing parameter coverage requires better information about parameters and their possible values. Manually selecting each method and analyzing its parameters is tedious. Tighter coupling between the IDL and the analysis is needed. If parameters with continuous ranges are to be supported, then ways of 
evaluating their coverage must be developed and automated.

The work with active probing has just started, but it seems a promising approach. It appears to broaden a tester's understanding of communication issues. More importantly, it provides a new dimension to testing activities. We believe active probing requires further study.

In summary, we are confident that one can gain insight and understanding about distributed system operation by remote procedure-call observation. Testers can use this knowledge to improve system test cases. Additionally, this information can be used as a measure of system test quality.

\section{Acknowledgments}

This research has been in part funded by the Stiftelsen för Kunskaps- och Kompetensutveckling through their Industrial Graduate School Program. Special thanks go to Henrik Samuelsson and Lars Rundberg of Ericsson $\mathrm{AB}$ in Stockholm for allowing us to observe the CIF application. Finally, we would like to thank Carl Rollo for proofreading.

\section{References}

[1] Ahlberg, C., Shneiderman, B. (1994) "Visual information seeking: tight coupling of dynamic query filters with starfield displays". Proc. of CHI'94, ACM Conference on Human Factors in Computing Systems, Boston, MA, April 24-28, 313-317.

[2] Auguston, M., Fritzson, P., (1996) "Parforman - an assertion language for specifying behaviour when debugging parallel applications". International Journal of Software Engineering and Knowledge Engineering, 6(4), Dec 1996, 609-640.

[3] Cain, J. W., McCrindle, R. J. (1999) "Software visualisation using $\mathrm{C}++$ lenses". Proc. of the $7^{\text {th }}$ International Workshop on Program Comprehension, Pittsburgh, PA, May 5-7.

[4] Cailleteau, L. (1999) "Interfaces for visualizing multivalued attributes: design and implementation using starfield displays'. Human-Computer Interaction Laboratory, University of Maryland, Tech. Report HCIL-99-20 (ftp://ftp.cs.umd.edu/pub/hcil/ Reports-AbstractsBibliography/99-20html/99-20.pdf).

[5] Comer, D., E., Lin, J., C., (1994) "Probing TCP Implementations". Proc. of the 1994 Summer USENIX Conference, Boston, MA, 245-255.

[6] The Computer Measurement Group's ARM Working Group, http://regions.cmg.org/regions/cmgarmw/ index.html, accessed February 11, 2002.

[7] De Pauw, W., Kimelman, D., Vlissides, J. (1994) "Modeling object-oriented program execution". Proc. of the $8^{\text {th }}$ European Conference on Object-Oriented Programming (ECOOP '94), Bologna, Italy, July 4-8, 163-182.

[8] Eick, S. G., Steffen, J. L., Sumner, E. E. (1992) "SeeSoft a tool for visualising line oriented software statistics".
IEEE Transactions on Software Engineering, 18(11), 957968.

[9] Fewster, M., Graham, D. (1999), "Software Test Automation: Effective use of test execution tools". ACM Press, ISBN 0-201-33140-3

[10] Ghosh, S., Mathur, A. P., (1999) "Issues in testing distributed component-based systems". Proc. of the First International ICSE Workshop on Testing Distributed Component Based Systems, Los Angeles, CA, May 17.

[11] Jacobson, I., Christerson, M., Jonsson, P., Övergaard, G. (1992) "Object-Oriented Software Engineering: A Use Case Driven Approach", Addison-Wesley, ISBN 0-20154435-0.

[12] Knight, C., Munro, M. (1999) "Comprehension with[in] virtual environment visualizations". Proc. of the $7^{\text {th }}$ International Workshop on Program Comprehension, Pittsburgh, PA, May 5-7.

[13] Larus, J. R., (1999) “Whole program paths". Proc. of the ACM SIGPLAN '99 Conference on Programming Language Design and Implementation, Atlanta, GA, May 1-4, 259-269.

[14] Malaiya, Y., K., Li, N., Karcich R., Skbbe, B., (1994) "The relationship between test coverage and reliability". Proc. International Symposium On Software Reliability Engineering, Nov, 186-195.

[15] von Mayrhauser, A., Vans, A. M., (1998) "Program Understanding Behavior During Adaptation of Large Scale Software". 6th International Workshop on Program Comprehension, Ischia, Italy, June 24-26, pp. 164-172

[16] Moe, J., Sandahl, K., (2002) "Using execution trace data to improve distributed systems: a replication". Proc. of the IEEE International Conference on Software Maintenance (ICSM 2002), 3-6 Oct., Montréal, Canada.

[17] Moe, J. Carr, D., (2002) "Using execution trace data to improve distributed systems", Software - Practice \& Experience, 32(9), 25 July 2002, pp. 889-906.

[18] Moe, J. Carr, D., (2001) 'Understanding distributed sy stems via Execution trace data". Proc. of the Ninth International Workshop on Program Comprehension, Toronto, Canada. 12-13 May, 60-67.

[19] Moore, I., (2001) “Jester - A unit tester”. At XP 2001, Villasimius, Sardinia

[20] Musa, J. D., (1993) "Operational profiles in software reliability engineering". IEEE Software, 10(2), 14-32.

[21] Object Management Group (1998) "The Common Object Request Broker: Architecture and Specification Revision 2.2". Framingham, MA, USA.

[22] Schauer, R., Keller, R. K. (1998) "Pattern visualisation for software comprehension". Proc. of the $6^{\text {th }}$ International Workshop on Program Comprehension, Ischia, Italy, June 24-26.

[23] Seshan, S., Steem, M., Katz, R., H., (1997) "SPAND: Shared Passive Network Performance Discovery". In Proc. of the USENIX Symposium on Internet Technologies and Systems, Monterey, CA, June.

[24] Walker, R. J., Murphy, G. C., Freeman-Benson, B., Wright, D., Swanson, D., Isaak, J. (1998) "Visualizing dy namic software system information through high-level models". Proc. of OOPSLA'98, Vancouver, Canada, Oct 18-22, 271-283. 
